\title{
A NON-NONSTANDARD PROOF OF REIMERS' EXISTENCE RESULT FOR HEAT SPDES
}

\author{
HASSAN ALLOUBA \\ Duke University, Mathematics Department \\ Durham, NC 27708 USA \\ E-mail: allouba@math.duke.edu
}

(Received November, 1996; Revised July, 1997)

In 1989, Reimers gave a nonstandard proof of the existence of a solution to heat SPDEs, driven by space-time white noise, when the diffusion coefficient is continuous and satisfies a linear growth condition. Using the martingale problem approach, we give a non-nonstandard proof of this fact, and with the aid of Girsanov's theorem for continuous orthogonal martingale measures (proved in a separate paper by the author), the result is extended to the case of a measurable drift.

Key words: Stochastic PDEs, Stochastic Heat Equation, Space-time White Noise, Martingale Problem.

AMS subject classifications: $60 \mathrm{H} 15,60 \mathrm{G} 48,60 \mathrm{G} 46$.

\section{Introduction}

We consider the SPDE

$$
\begin{gathered}
\frac{\partial U}{\partial t}=\frac{1}{2} \frac{\partial^{2} U}{\partial x^{2}}+a(U) \frac{\partial^{2} W}{\partial t \partial x} ; \quad(t, x) \in \mathbb{T} \times \mathbb{R}, \\
U(0, x)=h(x),
\end{gathered}
$$

where $\mathbb{T}:=[0, T]$ for some $0<T<\infty, W(t, x)$ is the Brownian sheet corresponding to the driving space-time white noise $\partial^{2} W / \partial t \partial x$, with intensity Lebesgue measure (see [15]). Our main result in this paper is

Theorem 1.1: Suppose that $a \in C(\mathbb{R} ; \mathbb{R})$ (continuous real-valued function on $\mathbb{R}$ ) and $h$ is a deterministic function in $C_{c}(\mathbb{R} ; \mathbb{R})$ (continuous real-valued function on $\mathbb{R}$ with compact support). Suppose further that there exists a constant $K>0$ such that

$$
a^{2}(x) \leq K\left(1+x^{2}\right)
$$

for all $x \in \mathbb{R}$. Then, there exists a solution to the heat SPDE (1.1).

Our approach will be as follows:

- We approximate the SPDE in (1.1) by a sequence of Stochastic DifferentialDifference Equations (SDDEs) associated with interacting diffusion models and 
solve the SDDEs;

- We then derive bounds on moments of spatial and temporal differences of the solutions obtained in the previous step;

- From those bounds, we conclude the tightness of the sequence of solutions;

- We then extract a subsequential limit, which solves a martingale problem that is equivalent to (1.1).

Girsanov's theorem for space-time white noise may then be used (see [1] or [2]) to prove existence for

$$
\begin{gathered}
\frac{\partial H}{\partial t}=\frac{1}{2} \frac{\partial^{2} H}{\partial x^{2}}+b(H)+a(H) \frac{\partial^{2} W}{\partial t \partial s} ;(t, x) \in \mathbb{T} \times \mathbb{R} \\
H(0, x)=h(x)
\end{gathered}
$$

where $a$ and $h$ are as above, and the drift $b$ is a Borel measurable real-valued function on $\mathbb{R}$ such that the random field $X(t, x)=b(U(t, x)) / a(U(t, x))$ satisfies the Novikov condition:

$$
E\left[\left(\exp \frac{1}{2} \int_{[0, t] \times \mathbb{R}} X^{2}(s, x) d s d x\right)\right]<\infty ; t \in \mathbb{T}
$$

where $U$ is a solution to the SPDE with no drive (1.1). For the convenience of the reader, we restate below Girsanov's theorem for white noise as well as the existence theorem relating (1.1) to (1.3). The reader is referred to [1] or [2] for more details.

Theorem 1.2: Let $\lambda$ be Lebesgue measure on the Borel $\sigma$-field $\mathfrak{B}(\mathbb{R})$ and $\mathscr{B}:=$ $\{A \in \mathscr{B}(\mathbb{R}) \mid \lambda(A)<\infty\}$. Let $W=\left\{W_{t}(A), \mathscr{F}_{t}: 0 \leq t<\infty, A \in \mathscr{B}\right\}$ be a space-time white noise $\underset{\sim}{o}$ a filtered probability space $\left(\Omega, \mathcal{F},\left\{\mathcal{F}_{t}\right\}_{t \geq 0}, P\right)$ (see [2] or [1]). Define the process $\widetilde{W}=\left\{\widetilde{W}_{t}(A), \mathscr{F}_{t} ; 0 \leq t<\infty, A \in \mathscr{B}_{0}\right\}$ by

$$
\tilde{W}_{t}(A):=W_{t}(A)-\int_{[0, t] \times A} Z(s, x) d s d x
$$

where $Z$ is some predictable random field (see [15]). Suppose that $Z$ satisfies (1.4). Then, for each fixed $T \in[0, \infty)$ and $A \in \Re$, the process $W=\left\{\widetilde{W}_{t}(B), \mathcal{F}_{t} ; 0 \leq t \leq T\right.$, $\left.B \in \mathscr{R}_{\mid A}\right\}$ is a space-time white noise on the probability space $\left(\Omega, \mathscr{F}_{T}, Q_{T}^{A}\right)$ having the intensity Lebesgue measure, where $\mathscr{B}_{\mid A}=\{B \in \mathscr{R} \mid B \subseteq A\}$ and $Q_{T}^{A}$ is the probability measure whose Radon-Nikodym derivative is given by

$$
\frac{d Q_{T}^{A}}{d P}=\exp \left[\int_{[0, T] \times A} Z(s, x) W(d s, d x)-\frac{1}{2} \int_{[0, T] \times A} Z^{2}(s, x) d s d x\right] .
$$

Theorem 1.3: Suppose that (1.1) has a solution $U$ and assume that $X$, as defined above, satisfies Novikov condition (1.4). Then, there exists a solution to (1.3).

There are two rigorous formulations for (1.1): the test function formulation and the Green's function formulation. The test function formulation is given by

$$
(U(T)-h, \varphi)=\frac{1}{2} \int_{0}^{t}\left(U(s), \varphi^{\prime \prime}\right) d s+\int_{\mathbb{R}} \int_{0}^{t} a(U(s, x)) \varphi(x) W(d s, d x)
$$


for all $t \in \mathbb{T}$ and $\varphi \in C_{c}^{\infty}(\mathbb{R} ; \mathbb{R})$, where $(\cdot, \cdot)$ denotes the scalar product on $L^{2}(\mathbb{R})$, and $C_{c}^{\infty}(\mathbb{R} ; \mathbb{R})$ is the space of infinitely differentiable functions with compact support. The Green's function formulation is the integral formulation

$$
U(t, x)=\int_{\mathbb{R}} G_{t}(x, y) h(y) d y+\int_{\mathbb{R}} \int_{0}^{t} G_{t-s}(x, y) a(U(s, y)) W(d s, d y) ;
$$

for all $t \in \mathbb{T}, x \in \mathbb{R}$, where $G_{t}(x, y)$ is the fundamental solution of the heat equation in $\mathbb{T} \times \mathbb{R}$. It is a well-known fact (see a discussion in [15] pp. 312-321), that the formulations in (1.6) and (1.7) are equivalent, provided the random field $a(U)$ is locally bounded, which we will assume throughout this article.

Remark 1.4: We will sometimes place a superscript $R_{i}, i=1,2, \ldots$ above a mathematical relation; e.g., $\stackrel{R_{1}}{\leq}$. This makes it easy to refer to the relation in question and renders our explanations more concise. Also, throughout this article, $K$ will denote a constant that may change its value from line to line.

\section{The Interacting Diffusion Models}

Consider the sequence of sets $\left(\mathbb{X}_{n}\right)_{n=1}^{\infty}$ defined by

$$
\mathbb{X}_{n}:=\left\{\ldots,-2 \delta_{n},-\delta_{n}, 0, \delta_{n}, 2 \delta_{n}, \ldots\right\}
$$

where $\delta_{n}>0$ for all $n$ and $\delta_{n} \underset{n \rightarrow \infty}{\rightarrow} 0$. Then the SPDE in (1.1) may be approximated by the following sequence of stochastic differential-difference equations (SDDEs):

$$
d \widetilde{U}_{n}^{x}(t)=\frac{1}{2} \Delta_{n} \widetilde{U}_{n}^{x}(t) d t+a\left(\widetilde{U}_{n}^{x}(t)\right) \frac{d W_{n}^{x}(t)}{\sqrt{\delta_{n}}},
$$

where $t \in \mathbb{T}$ and $x \in \mathbb{X}_{n}$, and $\Delta_{n} f(x)$ is the $n$th approximate Laplacian given by

$$
\Delta_{n} f(x):=\frac{f\left(x+\delta_{n}\right)-2 f(x)+f\left(x-\delta_{n}\right)}{\delta_{n}^{2}} .
$$

We think of $W_{n}^{x}(t)$ as a sequence of standard Brownian motions indexed by $x \in \mathbb{X}_{n}$, and we assume that, for each $n=1,2, \ldots, \widetilde{U}_{n}^{x}(0)=h(x)$ for all $x \in \mathbb{X}_{n}$. It follows from the boundedness of $h$ that

$$
\sup _{x \in \mathbb{X}_{n}}\left|\tilde{U}_{n}^{x}(0)\right| \leq K
$$

By a straightforward adaptation of Reimers' observations ([11], pp. 325-326) we get

Lemma 2.1: There is a solution $\widetilde{U}_{n}^{x}(t)$ to (2.1) satisfying

$$
\tilde{U}_{n}^{x}(t)=\sum_{y \in \mathbb{X}_{n}} \int_{0}^{t} Q_{\delta_{n}}^{x-y}(t-s) \frac{a\left(\tilde{U}_{n}^{y}(s)\right)}{\sqrt{\delta_{n}}} d W_{n}^{y}(s)+\sum_{y \in \mathbb{X}_{n}} Q_{\delta_{n}}^{x-y}(t) \widetilde{U}_{n}^{y}(0)
$$

where $Q_{\delta_{n}}^{x}(t)$ is the density of a random walk on the lattice $\mathbb{X}_{n}$, in which the times between transitions are exponentially distributed with mean $2 p \delta_{n}^{2}$, where $p$ is the pro- 
bability of a transition to the right (or to the left) and $1-2 p$ is the probability of no transition at a transition epoch. The subscript $\delta_{n}$ in $Q_{\delta_{n}}^{x}(t)$ is to remind us that the size of each step is $\delta_{n}$. The second term on the r.h.s. of (2.3) is deterministic and will henceforth be denoted by $\bar{U}_{n}^{x}(t)$. From (2.2), it follows that

$$
\left|\bar{U}_{n}^{x}(t)\right| \leq K \text {. }
$$

Remark 2.2: Equation (2.3) may be thought of as the discrete-space-continuous time "Green's function formulation" of the SDDE in (2.1).

\section{Some Bounds}

Here, we give bounds on the moments of spatial and temporal differences of the sequence $\left\{\widetilde{U}_{n}^{x}(t)\right)_{n=1}^{\infty}$ that are used to conclude tightness for our approximating sequence, along with some inequalities related to $Q_{\delta_{n}}^{x}(t)$ and some bounds on the moments of $\tilde{U}_{n}^{x}(t)$ that are useful in proving these spatial and temporal bounds. Since all the results in this section hold for all $n$, we will suppress the dependence on $n$ to simplify the notation. This section is a simple adaptation of Reimers' corresponding results to our setting, and most of the proofs will be omitted.

\subsection{Bounds related to $Q_{\delta}^{x}(t)$}

Lemma 3.1: There is a constant $K$ such that

$$
\sum_{x \in \mathbb{X}}\left(Q_{\delta}^{x}(t)\right)^{2} \leq K \delta / \sqrt{t}
$$

Lemma 3.2: There is a constant $K$ such that

$$
\int_{0}^{t} \sum_{x \in \mathbb{X}}\left(Q_{\delta}^{x}(s)\right)^{2} d s \leq K \delta \sqrt{t}
$$

Lemma 3.3: There is a constant $K$ such that

$$
\int_{0}^{t} \sum_{x \in \mathbb{X}}\left(Q_{\delta}^{x}(s)-Q_{\delta}^{x+z}(s)\right)^{2} d s \leq K \delta|z| .
$$

Lemma 3.4: There is a constant $K$ such that

$$
\int_{0}^{t} \sum_{x \in \mathbb{X}}\left(Q_{\delta}^{x}(t-s)-Q_{\delta}^{x}(r-s)\right)^{2} d s \leq K \delta \sqrt{t-r}
$$

for $r>t$, and with the convention that $Q_{b}^{x}(t)=0$ if $t<0$. 


\subsection{Bounds on moments of $\widetilde{U}^{x}(t)$}

Lemma 3.5: There exists a constant $K$ depending only on $p, q, \max _{x}\left|\tilde{U}^{x}(0)\right|$, and $T$ such that

$$
F_{q}(t) \leq K\left(1+\int_{0}^{t} \frac{F_{q}(s)}{\sqrt{t-s}} d s\right) \forall t \in \mathbb{T}
$$

where $F_{q}(t)=\sup _{x} E\left|\tilde{U}^{x}(t)\right|^{2 q}$.

Proof: Fix $q>1$, we then have:

$$
\begin{aligned}
& E\left|\widetilde{U}^{x}(t)\right|^{2 q}=E\left|\sum_{y \in \mathbb{X}} \int_{0}^{t} Q_{\delta}^{x-y}(t-s) \frac{a\left(\tilde{U}^{y}(s)\right)}{\sqrt{\delta}} d W^{y}(s)+\bar{U}^{x}(t)\right|^{2 q} \\
& \leq K E\left|\sum_{y \in \mathbb{X}} \int_{0}^{t} Q_{\delta}^{x-y}(t-s) \frac{a\left(\tilde{U}^{y}(s)\right)}{\sqrt{\delta}} d W^{y}(s)\right|^{2 q}+K\left|\bar{U}^{x}(t)\right|^{2 q}
\end{aligned}
$$

Now, as in [11] p. 327, applying Burkholder inequality to

we get

$$
V^{x}(t)=\sum_{y \in \mathbb{X}} \int_{0}^{t} Q_{\delta}^{x-y}(t-s) \frac{a\left(\tilde{U}^{y}(s)\right)}{\sqrt{\delta}} d W^{y}(s)
$$

$$
E\left|V^{x}(t)\right|^{2 q} \leq K E\left|\sum_{y \in \mathbb{X}} \int_{0}^{t}\left(Q_{\delta}^{x-y}(t-s)\right)^{2} \frac{a^{2}\left(\tilde{U}^{y}(s)\right)}{\delta} d s\right|^{q}
$$

so that (3.2) reduces to

$$
E\left|\tilde{U}^{x}(t)\right|^{2 q} \leq K E\left|\sum_{y \in \mathbb{X}} \int_{0}^{t}\left(Q_{\delta}^{x-y}(t-s)\right)^{2} \frac{a^{2}\left(\tilde{U}^{y}(s)\right)}{\delta} d s\right|^{q}+K\left|\bar{U}^{x}(t)\right|^{2 q} .
$$

Now, for a fixed point $(t, x) \in \mathbb{T} \times \mathbb{X}$, let $\mu_{t}^{x}$ be the measure on $[0, t] \times \mathbb{X}$ defined by $d \mu_{t}^{x}(s, y)=\left(\left(Q_{\delta}^{x-y}(t-s)\right)^{2} / \delta\right) d s$, and let $\left|\mu_{t}^{x}\right|=\mu_{t}^{x}([0, t] \times \mathbb{X})$. Then, (3.3) can be rewritten as

$$
E\left|\tilde{U}^{x}(t)\right|^{2 q} \leq K E\left|\int_{[0, t] \times \mathbb{X}} a^{2}\left(\tilde{U}^{y}(s)\right) \frac{d \mu_{t}^{x}(s, y)}{\left|\mu_{t}^{x}\right|}\right|^{q}\left|\mu_{t}^{x}\right|^{q}+K\left|\bar{U}^{x}(t)\right|^{2 q} .
$$

Observing that $\mu_{t}^{x} /\left|\mu_{t}^{x}\right|$ is a probability measure, we apply Jensen's inequality to (3.4) to obtain

$$
E\left|\widetilde{U}^{x}(t)\right|^{2 q} \leq K E\left[\int_{[0, t] \times \mathbb{X}}\left|a\left(\tilde{U}^{y}(s)\right)\right|^{2 q} \frac{d \mu_{t}^{x}(s, y)}{\left|\mu_{t}^{x}\right|}\right]\left|\mu_{t}^{x}\right|^{q}+K\left|\bar{U}^{x}(t)\right|^{2 q}
$$




$$
\begin{aligned}
& =K\left[\int_{[0, t] \times \mathbb{X}} E\left|a\left(\tilde{U}^{y}(s)\right)\right|^{2 q} d \mu_{t}^{x}(s, y)\right]\left|\mu_{t}^{x}\right|^{q-1}+K\left|\bar{U}^{x}(t)\right|^{2 q} \\
=K & {\left[\sum_{y \in \mathbb{X}} \int_{0}^{t} E\left|a\left(\tilde{U}^{y}(s)\right)\right|^{2 q} \frac{\left(Q_{\delta}^{x-y}(t-s)\right.}{\delta} d s\right]\left|\mu_{t}^{x}\right|^{q-1}+K\left|\bar{U}^{x}(t)\right|^{2 q} . }
\end{aligned}
$$

Now, by Lemma 3.2, $\left|\mu_{t}^{x}\right|^{q-1}$ is uniformly bounded for $t \leq T$. This, together with (2.4) give us

$$
\begin{aligned}
& E\left|\tilde{U}^{x}(t)\right|^{2 q} \leq K\left(1+\sum_{y \in \mathbb{X}} \int_{0}^{t} E\left|a\left(\tilde{U}^{y}(s)\right)\right|^{2 q} \frac{\left(Q_{\delta}^{x-y}(t-s)\right)^{2}}{\delta} d s\right) \\
& \stackrel{R_{1}}{\leq} K\left(1+\sum_{y \in \mathbb{X}} \int_{0}^{t} E\left|1+\left(\tilde{U}^{y}(s)\right)^{2}\right|^{q} \frac{\left(Q_{\delta}^{x-y}(t-s)\right)^{2}}{\delta} d s\right) \\
& \leq K\left(1+\sum_{y \in \mathbb{X}} \int_{0}^{t}\left(1+\mid E\left(\tilde{U}^{y}(s) \mid\right)^{2 q}\right) \frac{\left(Q_{\delta}^{x-y}(t-s)\right)^{2}}{\delta} d s\right) \\
& \stackrel{R_{2}}{\leq} K\left(1+\sum_{y \in \mathbb{X}} \int_{0}^{t} E\left|\left(\tilde{U}^{y}(s)\right)\right|^{2 q} \frac{\left(Q_{\delta}^{x-y}(t-s)\right)^{2}}{\delta} d s\right) \\
& =K\left(1+\int_{0}^{t} \sum_{y \in \mathbb{X}} E\left|\left(\tilde{U}^{y}(s)\right)\right|^{2 q} \frac{\left(Q_{\delta}^{x-y}(t-s)\right)^{2}}{\delta} d s\right) .
\end{aligned}
$$

Here $R_{1}$ follows from the linear growth condition on $a$ and $R_{2}$ is a consequence of Lemma 3.2. Now, letting $F_{q}(s)=\sup _{x} E\left|\widetilde{U}^{x}(s)\right|^{2 q}$, we get

$$
\begin{aligned}
E\left|\tilde{U}^{x}(t)\right|^{2 q} \leq & K\left(1+\int_{0}^{t} F_{q}(s) \sum_{y \in \mathbb{X}} \frac{\left(Q_{\delta}^{x-y}(t-s)\right)^{2}}{\delta} d s\right) \\
& R_{3} K\left(1+\int_{0}^{t} \frac{F_{q}(s)}{\sqrt{t-s}} d s\right)
\end{aligned}
$$

Here, $R_{3}$ follows from Lemma 3.1. this implies that

We easily obtain:

$$
F_{q}(t) \leq K\left(1+\int_{0}^{t} \frac{F_{q}(s)}{\sqrt{t-s}} d s\right) .
$$

Corollary 3.6: There exists a constant $K$ such that 


$$
F_{q}(t) \leq K\left(1+\int_{0}^{t} F_{q}(s) d s\right)
$$

for all $t \in \mathbb{T}$.

Proof: Iterating the bound in Lemma 3.5 once, and changing the order of integration, we obtain

$$
\begin{gathered}
F_{q}(t) \leq K\left\{1+K\left[\int_{0}^{t} \frac{1}{\sqrt{t-s}} d s+\int_{0}^{t} F_{q}(r)\left(\int_{r}^{t} \frac{1}{\sqrt{t-s}} \frac{1}{\sqrt{s-r}} d s\right) d r\right]\right\} \\
\leq K\left\{1+K\left[2 \sqrt{T}+\int_{0}^{t} F_{q}(r)\left(\int_{0}^{T} \frac{1}{\sqrt{T-s}} \frac{1}{\sqrt{s}} d s\right) d r\right]\right\} \\
\leq K\left\{1+\int_{0}^{t} F_{q}(r) d r\right\}
\end{gathered}
$$

By Gronwall's Lemma we have:

Lemma 3.7: There exists a constant $K$ depending only on $p, q, \max _{x}\left|\tilde{U}^{x}(0)\right|$, and $T$ such that

$$
E\left|\tilde{U}^{x}(t)\right|^{2 q} \leq K \exp (K t)
$$

for all $t \in \mathbb{T}$ and all $x \in \mathbb{X}$.

\subsection{Bounds on moments of spatial and temporal differences}

Let $\widetilde{U}^{x}(t)=V^{x}(t)+\bar{U}^{x}(t)$, where $V$ denotes the first term on the r.h.s. of (2.3) (the random term). Using the inequalities of the previous two subsections, we obtain:

Lemma 3.8: (Spatial Differences) There exists a constant $K$ depending only on $p, q, \max _{x}\left|\widetilde{U}^{x}(0)\right|$, and $T$ such that

$$
E\left|V^{x}(t)-V^{y}(t)\right|^{2 q} \leq K|x-y|^{q},
$$

for all $x, y \in \mathbb{X}$ and for $t \in \mathbb{T}$.

Lemma 3.9: (Temporal Differences) There exists a constant $K$ depending only on $p, q, \max _{x}\left|\widetilde{U}^{x}(0)\right|$, and $T$ such that

$$
E\left|V^{x}(t)-V^{x}(r)\right|^{2 q} \leq K|t-r|^{q / 2}
$$

for all $x \in \mathbb{X}$ and for all $t, r \in \mathbb{T}$.

\section{The Sequence of SDDEs Solutions is Tight}

Let $\widetilde{\widetilde{U}}_{n}(t, x)$ be the extension of $\widetilde{U}_{n}^{x}(t)$ to $\mathbb{T} \times \mathbb{R}$ obtained by linear interpolation of the $\widetilde{U}_{n}^{x}(t)$ 's between the lattice points of $\mathbb{X}_{n}$. The following is Kolmogorov's continuity criterion for random fields. (See [9] pp. 53-55 and p. 118; see also Corollary 1.2 in [15].)

Lemma 4.1: Suppose $\left\{X(t) ; t \in[0, T]^{d}\right\}, d \geq 2$, is a real-valued random field 
satisfying

$$
E|X(t)-X(s)|^{\alpha} \leq C\|t-s\|^{d+\beta}
$$

for some positive constants $\alpha, \beta$ and $C$, for some norm $\|\cdot\|$ on $\mathbb{R}^{d}$. Then there exists a continuous modification $\widetilde{X}=\left\{\widetilde{X}(t) ; t \in[0, T]^{d}\right\}$ of $X$.

We also have the following tightness criterion:

Lemma 4.2: For each $n=1,2, \ldots$, let $\left\{X_{n}(t) ; t \in \mathbb{T} \times \mathbb{R}\right\}$ be a real-valued continuous random field. The sequence $\left\{X_{n}\right\}$, regarded as a sequence of random variables taking values in $C(\mathbb{T} \times \mathbb{R})$, is tight in $C(\mathbb{T} \times \mathbb{R})$ if:

(i) $\quad\left\{X_{n}(0)\right\}$ is tight,

(ii) $E\left|X_{n}(t)-X_{n}(s)\right|^{\alpha} \leq C\|t-s\|^{2+\beta}$, for some positive constants $\alpha, \beta, C$.

The proof of Lemma 4.2 follows very closely the proof of Theorem 12.3 in [3] and will not be repeated.

Remark 4.3: Since all norms on $\mathbb{R}^{d}$ are equivalent, the norm $\|\cdot\|$ in Lemma 4.1 and Lemma 4.2 may be chosen to be any norm on $\mathbb{R}^{d}$.

Lemma 4.4: For each $n$, the random field $\widetilde{\widetilde{U}}_{n}=\left\{\widetilde{\widetilde{U}}_{n}(t, x) ; t \in \mathbb{T}, x \in \mathbb{R}\right\}$ has a continuous modification, which we will also denote by $\widetilde{\widetilde{U}}_{n}$, and the sequence $\left(\widetilde{\widetilde{U}}_{n}\right)_{n=1}^{\infty}$ is tight in $C(\mathbb{T} \times \mathbb{R})$.

Proof: This follows from Lemma 3.8, Lemma 3.9, Lemma 4.1, and Lemma 4.2 (see also the discussion on p. 97 in [3]). This is a routine argument, so we omit the details.

\section{The Martingale Problem}

Since the sequence $\left(\widetilde{\widetilde{U}}_{n}(t, x)\right)_{n=1}^{\infty}$ is tight, it follows that there exists a subsequence $\widetilde{\widetilde{U}}_{n_{k}}$ which induces measures $\widetilde{\widetilde{P}}_{n_{k}}$ on $(C, \mathrm{C})$ that converge weakly to a limit $\widetilde{\widetilde{P}}$, where $C:=C(\mathbb{T} \times \mathbb{R} ; \mathbb{R})$ and $\mathcal{C}:=\mathscr{B}(C)$, where $\mathscr{B}(C)$ is the Borel $\sigma$-field over $C$. Now, following Skorokhod [13], we can construct processes $Y_{k} \stackrel{d}{=} \widetilde{\widetilde{U}}_{n_{k}}$ on some probability space $\left(\Omega^{S}, \mathscr{F}^{S}, P^{S}\right)$ such that with probability 1 , as $k \rightarrow \infty, Y_{k}(t, x)$ converges to a random field $Y(t, x)$ uniformly on compact subsets of $\mathbb{T} \times \mathbb{R}$ for any $T<\infty$. We will show that $Y(t, x)$ is a solution to the heat SPDE (1.1) by solving an equivalent martingale problem ([8]).

For every $\varphi \in C_{c}^{\infty}(\mathbb{R} ; \mathbb{R})$, let

$$
S^{\varphi}\left(Y_{k}, t\right)=\sum_{x \in \mathbb{X}_{n_{k}}} Y_{k}(t, x) \varphi(x) \delta_{n_{k}}-\frac{1}{2} \int_{0}^{t} \sum_{x \in \mathbb{X}_{n_{k}}} Y_{k}(s, x) \Delta_{n_{k}} \varphi(x) \delta_{n_{k}} d s
$$

and let $\mathcal{G}_{t}$ be the filtration on $\left(\Omega^{S}, \mathscr{F}^{S}, P^{S}\right)$ generated by the process $S^{\varphi}\left(Y_{k}, t\right)$ for all $\varphi$ and all $k$; i.e., $\mathcal{G}_{t}=\sigma\left[S^{\varphi}\left(Y_{k}, s\right) ; 0 \leq s \leq t, \varphi \in C_{c}^{\infty}(\mathbb{R} ; \mathbb{R}), k=1,2, \ldots\right]$. We prove Theorem 1.1 by proving:

Theorem 5.1: There exists an extension $\left(\widetilde{\Omega}^{S}, \tilde{\mathfrak{F}}^{S},\left\{\tilde{\mathfrak{G}}_{t}\right\}_{t} \geq 0, P^{S}\right)$ of the filtered probability space $\left(\Omega^{S}, \mathcal{F}^{S},\left\{\mathfrak{G}_{t}\right\}_{t>0}, P^{S}\right)$ and a white noise $W$ defined on it such that the pair $(Y, W)$ solves $S P D E(1.1)$ on $\left(\widetilde{\Omega}^{S}, \widetilde{\mathscr{F}}^{S},\left\{\widetilde{\mathscr{G}}_{t}\right\}_{t \geq 0}, P^{S}\right)$. 
The proof of Theorem 5.1 follows from Theorem 3.3 in [8] in conjunction with Theorem 5.2 and Theorem 5.3 below.

Theorem 5.2: For $\varphi \in C_{c}^{\infty}(\mathbb{R} ; \mathbb{R})$, we have

(i) $\quad\left\{S^{\varphi}(t), \mathfrak{g}_{t}\right\}$ is a martingale, for every $\varphi \in C_{c}^{\infty}(\mathbb{R} ; \mathbb{R})$, where

$$
S^{\varphi}(t):=(Y(t), \varphi)-\frac{1}{2} \int_{0}^{t}\left(Y(s), \varphi^{\prime \prime}\right) d s,
$$

where $(\cdot, \cdot)$ denotes the scalar product on $L^{2}(\mathbb{R})$,

(ii) $\langle(Y, \varphi)\rangle_{t}=\int_{0}^{t} \int_{\mathbb{R}} a^{2}(Y(s, x)) \varphi^{2}(x) d x d s$.

Proof: (i) Assume that the sequence of Brownian motions $W_{n}^{x}(t)$ in $(2.1)$ is defined on some probability space $(\Omega, \mathcal{F}, P)$ and adapted to a filtration $\left\{\mathscr{F}_{t}\right\}_{t} \geq 0$. We first observe that for any $k$,

$$
\tilde{U}_{n_{k}}^{x}(t) \varphi(x) \delta_{n_{k}}-\frac{1}{2} \int_{0}^{t} \Delta_{n_{k}} \tilde{U}_{n_{k}}^{x}(t) \varphi(x) \delta_{n_{k}} d s
$$

is an $\mathscr{F}_{t}$-martingale for each $x \in \mathbb{X}_{n_{k}}$. This is obvious from (2.1). Now since $\varphi$ has a compact support, it follows that

$$
\begin{gathered}
\sum_{x \in \mathbb{X}_{n_{k}}} \tilde{U}_{n_{k}}^{x}(t) \varphi(x) \delta_{n_{k}}-\frac{1}{2} \int_{0}^{t} \sum_{x \in \mathbb{X}_{n_{k}}} \Delta_{n_{k}} \tilde{U}_{n_{k}}^{x}(s) \varphi(x) \delta_{n_{k}} d s \\
=\sum_{x \in \mathbb{X}_{n_{k}}} \tilde{U}_{n_{k}}^{x}(t) \varphi(x) \delta_{n_{k}}-\frac{1}{2} \int_{0}^{t} \sum_{x \in \mathbb{X}_{n_{k}}} \tilde{U}_{n_{k}}^{x}(s) \Delta_{n_{k}} \varphi(x) \delta_{n_{k}} d s \\
:=S^{\varphi}\left(\tilde{U}_{n_{k}}, t\right)
\end{gathered}
$$

is a finite sum, and hence an $\mathcal{F}_{t}$-martingale. Replacing the $\tilde{U}_{n_{k}}^{x}(t)$ 's in (5.2) by the $Y_{k}(t, x)$ 's and letting $k \rightarrow \infty$ we get that $S^{\varphi}\left(Y_{k}, t\right) \rightarrow S^{\varphi}(t)$ a.s. uniformly on $\mathbb{T}$. In addition, the $S^{\varphi}\left(Y_{k}, t\right)$ are uniformly integrable for each $t$ and each $\varphi$ (for each $t$ and each $\varphi \in C_{c}^{\infty}(\mathbb{R} ; \mathbb{R}), E\left|S^{\varphi}\left(Y_{k}, t\right)\right|^{p} \leq M_{p}<\infty \forall k$, for some constant $M_{p}$, for any $p>2$ ). So, if $s<t$,

$$
E\left[\left(S^{\varphi}(t)-S^{\varphi}(s)\right) \mid \mathfrak{g}_{s}\right]=\lim _{k \rightarrow \infty} E\left[\left(S^{\varphi}\left(Y_{k}, t\right)-S^{\varphi}\left(Y_{k}, s\right)\right) \mid \mathfrak{G}_{s}\right]=0 .
$$

This proves $(i)$.

(ii) From (2.1) it follows that

$$
\begin{gathered}
d\left[\sum_{x \in \mathbb{X}_{n_{k}}} \tilde{U}_{n_{k}}^{x}(t) \varphi(x) \delta_{n_{k}}\right]=\frac{1}{2} \sum_{x \in \mathbb{X}_{n_{k}}} \Delta_{n_{k}} \tilde{U}_{n_{k}}^{x}(t) \varphi(x) \delta_{n_{k}} d t \\
+\sum_{x \in \mathbb{X}_{n_{k}}} \frac{a\left(\widetilde{U}_{n_{k}}^{x}(t)\right)}{\sqrt{\delta_{n_{k}}}} \varphi(x) \delta_{n_{k}} d W_{n_{k}}^{x}(t)
\end{gathered}
$$




$$
=\frac{1}{2} \sum_{x \in \mathbb{X}_{n_{k}}} \tilde{U}_{n_{k}}^{x}(t) \Delta_{n_{k}} \varphi(x) \delta_{n_{k}} d t+\sum_{x \in \mathbb{X}_{n_{k}}} \frac{a\left(\tilde{U}_{n_{k}}^{x}(t)\right)}{\sqrt{\delta_{n_{k}}}} \varphi(x) \delta_{n_{k}} d W_{n_{k}}^{x}(t) .
$$

Observing that the first term on the right-hand side of the above equation is of bounded variation, and that the $\left(W_{n_{k}}^{x}(t)\right)_{x \in \mathbb{X}_{n_{k}}}$ is a sequence of independent Brown-
ian motions, we obtain

$$
\left\langle\sum_{x \in \mathbb{X}_{n_{k}}} \tilde{U}_{n_{k}}^{x} \varphi(x) \delta_{n_{k}}\right\rangle_{t}=\int_{0}^{t}\left[\sum_{x \in \mathbb{X}_{n_{k}}} a^{2}\left(\tilde{U}_{n_{k}}^{x}(s)\right) \varphi^{2}(x) \delta_{n_{k}}\right] d s
$$

Again, replacing the $\widetilde{U}_{n_{k}}^{x}(t)$ 's in (5.3) by the $Y_{k}(t, x)$ 's, we get, for $0 \leq r \leq t<\infty$,

$$
\begin{aligned}
& E\left[\left(\sum_{x \in \mathbb{X}_{n_{k}}} Y_{k}(t, x) \varphi(x) \delta_{n_{k}}-\sum_{x \in \mathbb{X}_{n_{k}}} Y_{k}(r, x) \varphi(x) \delta_{n_{k}}\right)^{2} \mid \mathfrak{g}_{r}\right] \\
& =E\left[\int_{r}^{t} \sum_{x \in \mathbb{X}_{n_{k}}} a^{2}\left(Y_{k}(s, x)\right) \varphi^{2}(x) \delta_{n_{k}} d s \mid g_{r}\right] \text {. }
\end{aligned}
$$

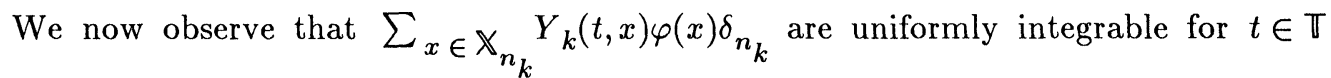
and for each $\varphi$ (again, for $t \in \mathbb{T}$ and for each $\varphi \in C_{c}^{\infty}(\mathbb{R} ; \mathbb{R}), E \mid \sum_{x \in \mathbb{X}_{n_{k}}} Y_{k}(t, x)$ $\times\left.\varphi(x) \delta_{n_{k}}\right|^{p} \leq K_{p}<\infty \forall k$, for some constant $K_{p}$, for any $\left.p>2\right)$. Consequently,

$$
\begin{aligned}
& {\left[\lim _{k \rightarrow \infty} E\left(\sum_{x \in \mathbb{X}_{n_{k}}} Y_{k}(t, x) \varphi(x) \delta_{n_{k}}-\sum_{x \in \mathbb{X}_{n_{k}}} Y_{k}(r, x) \varphi(x) \delta_{n_{k}}\right)^{2} \mid g_{r}\right]} \\
& =E\left[\left(\int_{\mathbb{R}} Y(t, x) \varphi(x) d x-\int_{\mathbb{R}} Y(r, x) \varphi(x) d x\right)^{2} \mid g_{r}\right] .
\end{aligned}
$$

Also, $a$ is continuous, and hence locally bounded. We see by the Dominated Convergence theorem that

$$
\begin{gathered}
\lim _{k \rightarrow \infty} E\left[\int_{r}^{t} \sum_{x \in \mathbb{X}_{n_{k}}} a^{2}\left(Y_{k}(s, x)\right) \varphi^{2}(x) \delta_{n_{k}} d s \mid \mathfrak{g}_{r}\right] \\
=E\left[\int_{r}^{t} \int_{\mathbb{R}} a^{2}(Y(s, x)) \varphi^{2}(x) d x d s \mid \mathfrak{g}_{r}\right] .
\end{gathered}
$$

Now, equations (5.4), (5.5) and (5.6) yield 


$$
\langle(Y, \varphi)\rangle_{t}=\int_{0}^{t} \int_{\mathbb{R}} a^{2}(Y(s, x)) \varphi^{2}(x) d x d s,
$$

and $(i i)$ is proved.

Let $\mathscr{D}:=\left\{f: C(\mathbb{R} ; \mathbb{R}) \rightarrow \mathbb{R} \mid f(X)=\tilde{f}\left(\left(X, \varphi_{1}\right), \ldots,\left(X, \varphi_{n}\right)\right), \quad\right.$ for $\quad$ some $\quad n \geq 1$, $\varphi_{1}, \ldots, \varphi_{n} \in C_{K}^{\infty}(\mathbb{R} ; \mathbb{R})$ and $\left.\tilde{f} \in C_{K}^{\infty}\left(\mathbb{R}^{n} ; \mathbb{R}\right)\right\}$, and define the operator $L$ with domain $\mathscr{D}$ as follows:

$$
\begin{aligned}
L f(X):=\frac{1}{2} \sum_{i, j=1}^{n} & \left\{\int_{\mathbb{R}} a^{2}(X(x)) \varphi_{i}(x) \varphi_{j}(x) d x\right\} D_{i j} \tilde{f}\left(\left(X, \varphi_{1}\right), \ldots,\left(X, \varphi_{n}\right)\right) \\
& +\frac{1}{2} \sum_{i}^{n}\left(X, \varphi_{i}^{\prime \prime}\right) D_{i} \tilde{f}\left(\left(X, \varphi_{1}\right), \ldots,\left(X, \varphi_{n}\right)\right) .
\end{aligned}
$$

We now prove our key martingale theorem.

Theorem 5.3: If $f \in \mathscr{D}$, then

$$
f(Y(t, \cdot))-\int_{0}^{t} L f(Y(s, \cdot)) d s \text { is a } \mathfrak{G}_{t} \text {-martingale. }
$$

Proof: Since $f \in \mathscr{D}$, we have that

$$
\begin{gathered}
f(Y(t, \cdot))-f(Y(0, \cdot)) \\
\stackrel{R_{1}}{=} \tilde{f}\left(\left(Y(t), \varphi_{1}\right), \ldots,\left(Y(t), \varphi_{n}\right)\right)-\tilde{f}\left(\left(Y(0), \varphi_{1}\right), \ldots,\left(Y(0), \varphi_{n}\right)\right) \\
\stackrel{R_{2}}{=} \sum_{i=1}^{n} \int_{0}^{t} D_{i} \tilde{f}\left(\left(Y(t), \varphi_{1}\right), \ldots,\left(Y(t), \varphi_{n}\right)\right) d\left(Y(s), \varphi_{i}\right) \\
+\frac{1}{2} \sum_{i, j=1}^{n} \int_{0}^{t} D_{i j} \tilde{f}\left(\left(Y(t), \varphi_{1}\right), \ldots,\left(Y(t), \varphi_{n}\right)\right) d\left\langle\left(Y, \varphi_{i}\right),\left(Y, \varphi_{j}\right)\right\rangle_{s},
\end{gathered}
$$

where $R_{1}$ follows for some $n \geq 1, \varphi_{1}, \ldots, \varphi_{n} \in C_{K}^{\infty}(\mathbb{R} ; \mathbb{R})$ and $\tilde{f} \in C_{K}^{\infty}\left(\mathbb{R}^{n}, \mathbb{R}\right)$, by the definition of $\mathscr{D}$; and $R_{2}$ follows from Itô's rule for $n$-continuous semimartingales.

However,

$$
\begin{gathered}
\left\langle\left(Y, \varphi_{i}\right),\left(Y, \varphi_{j}\right)\right\rangle_{s}=\frac{1}{4}\left\{\left\langle\left(Y, \varphi_{i}+\varphi_{j}\right)\right\rangle_{s}-\left\langle\left(Y, \varphi_{i}-\varphi_{j}\right)\right\rangle_{s}\right\} \\
\stackrel{R_{1}}{=} \int_{0}^{s} \int_{\mathbb{R}} a^{2}(Y(u, x)) \varphi_{i}(x) \varphi_{j}(x) d x d u \\
=\int_{0}^{s}\left(a^{2}(Y(u)), \varphi_{i} \varphi_{j}\right) d u
\end{gathered}
$$

where $R_{1}$ follows from part (ii) of Theorem 5.2. Also, by part ( $i$ ) of Theorem 5.2 , we have $d(Y(s), \varphi)=d S^{\varphi}(s)+\frac{1}{2}\left(Y(s), \varphi^{\prime \prime}\right) d s$, so that we can rewrite the expression for 
$f(Y(t, \cdot))-f(Y(0, \cdot))$ above as:

$$
\begin{gathered}
f(Y(t, \cdot))-f(Y(0, \cdot)) \\
=\frac{1}{2} \sum_{i=1}^{n} \int_{0}^{t} D_{i} \tilde{f}\left(\left(Y(s), \varphi_{1}\right), \ldots,\left(Y(s), \varphi_{n}\right)\right)\left(Y(s), \varphi_{i}^{\prime \prime}\right) d s \\
+\frac{1}{2} \sum_{i, j=1}^{n} \int_{0}^{t} D_{i j} \tilde{f}\left(\left(Y(s), \varphi_{1}\right), \ldots,\left(Y\left(s 0, \varphi_{n}\right)\right)\left\{\left(a^{2}(Y(s)), \varphi_{i} \varphi_{j}\right)\right\} d s\right. \\
+\frac{1}{2} \sum_{i=1}^{n} \int_{0}^{t} D_{i} \tilde{f}\left(\left(Y(s), \varphi_{1}\right), \ldots,\left(Y(s), \varphi_{n}\right)\right) d S^{\varphi_{i}}(s) .
\end{gathered}
$$

The third term on the right-hand side of (5.10) is a finite sum of $g_{t}$-martingales, by part $(i)$ of Theorem 5.2 and the boundedness of $D_{i} \tilde{f}$, and hence is a $g_{t}$-martingale. Now,

$$
\begin{aligned}
& \int_{0}^{t} L f(Y(s, \cdot)) d s=\frac{1}{2} \sum_{i=1}^{n} \int_{0}^{t} D_{i} \tilde{f}\left(\left(Y(s), \varphi_{1}\right), \ldots,\left(Y(s), \varphi_{n}\right)\right)\left(Y(s), \varphi_{i}^{\prime \prime}\right) d s \\
& \quad+\frac{1}{2} \sum_{i, j=1}^{n} \int_{0}^{t} D_{i j} \tilde{f}\left(\left(Y(s), \varphi_{1}\right), \ldots,\left(Y(s), \varphi_{n}\right)\right)\left\{\left(a^{2}(Y(s)), \varphi_{i} \varphi_{j}\right)\right\} d s
\end{aligned}
$$

Consequently,

$$
f(Y(t, \cdot))-f(Y(0, \cdot))-\int_{0}^{t} L f(Y(s, \cdot)) d s
$$

is a $g_{t}$-martingale. This completes the proof.

\section{Acknowledgement}

This paper is based on a part of my Ph.D. dissertation [1] under Rick Durrett. I would like to thank Rick for his constant encouragement and for the excellent scientific environment he provided for me while at Cornell. I wish to also thank an anonymous referee for his comments, which made this paper more readable.

\section{References}

[1] Allouba, H., Types of SPDEs: Existence, Uniqueness, and Girsanov's Theorem, Ph.D. Dissertation, Cornell University 1996.

[2] Allouba, H., Different types of SPDEs in the eyes of Girsanov's Theorem, Stoch. 
Anal. and Appl., to appear.

[3] Billingsley, P., Convergence of Probability Measures, John Wiley and Sons, New York 1968.

[4] DaPrato, G. and Zabczyk, J., Stochastic Equations in Infinite Dimensions, Cambridge University Press, Cambridge 1992.

[5] Durrett, R., Brownian Motion and Martingales in Analysis, Wadsworth Advanced Books and Software, California 1984.

[6] Durrett, R., Probability: Theory and Examples, Wadsworth and Brooks, California 1991.

[7] El Karoui, N. and Méléard, S., Martingale measures and stochastic calculus, Probab. Theory Relat. Fields 84 (1990), 83-101.

[8] Iwata, K., An infinite dimensional stochastic differential equation with state space $C(\mathbb{R})$, Probab. Theory Relat. Fields 74 (1987), 141-159.

[9] Karatzas, I. and Shreve, S., Brownian Motion and Stochastic Calculus, SpringerVerlag, New York 1988.

[10] Protter, P., Stochastic Integration and Differential Equations, Springer-Verlag, New York 1990.

[11] Reimers, M., One dimensional stochastic partial differential equations and the branching measure diffusion, Probab. Theory Relat. Fields 81 (1989), 319-340.

[12] Shiga, T., Two contrasting properties of solutions for one-dimensional stochastic partial differential equations, Can. J. Math. 46:2 (1994), 415-437.

[13] Skorokhod, A.V., Limit theorems for stochastic processes, TPA 1 (1956), 261290.

[14] Stroock, D.W. and Varadhan, S.R., Multidimensional Diffusion Processes, Springer-Verlag, New York 1979.

[15] Walsh, J.B., An Introduction to Stochastic Partial Differential Equations, In: École d'Été de Probabilités de Saint-Flour XIV-1984, Springer-Verlag, New York 1986. 


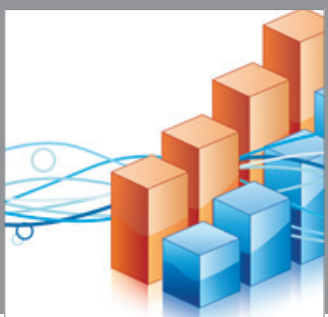

Advances in

Operations Research

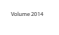

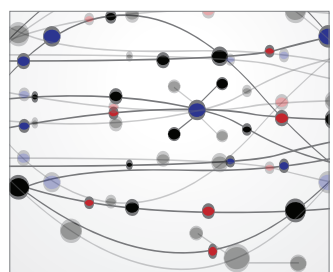

\section{The Scientific} World Journal
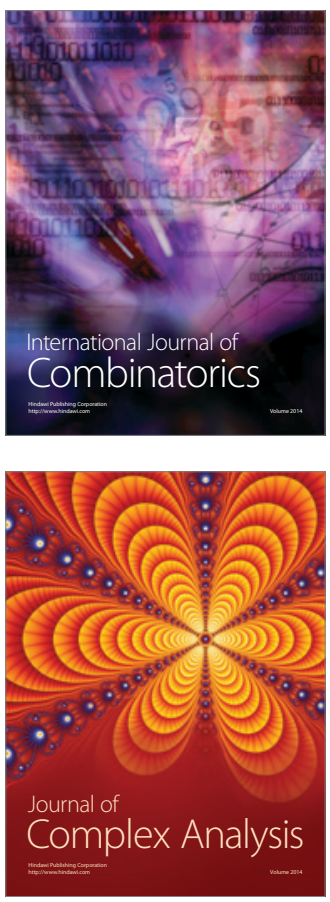

International Journal of

Mathematics and

Mathematical

Sciences
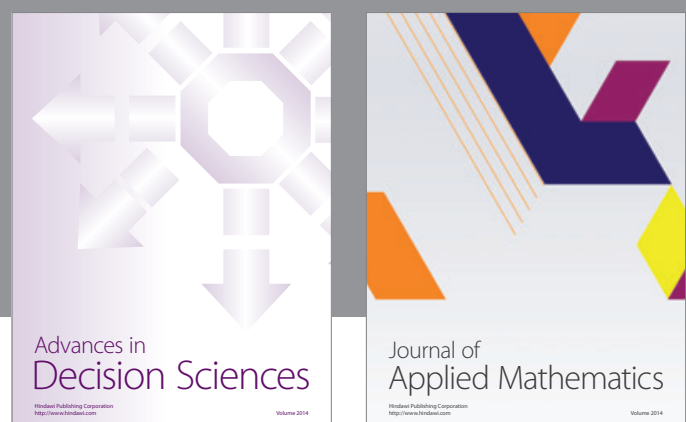

Journal of

Applied Mathematics
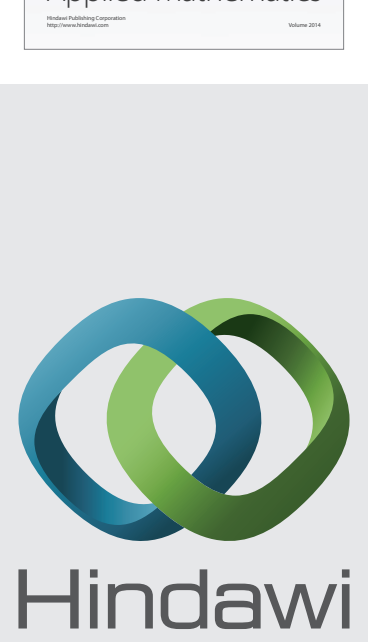

Submit your manuscripts at http://www.hindawi.com
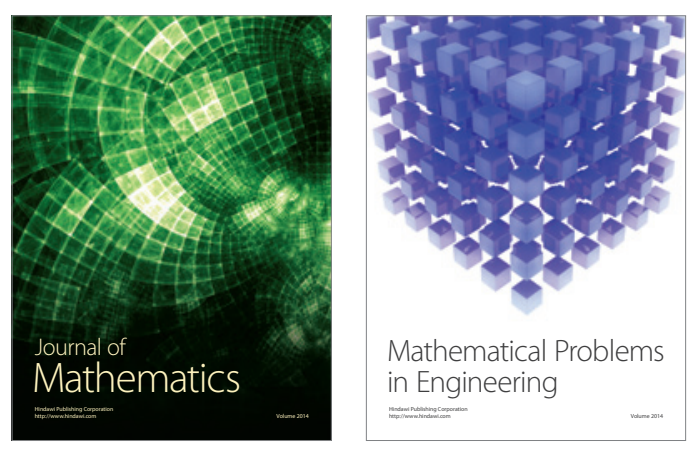

Mathematical Problems in Engineering
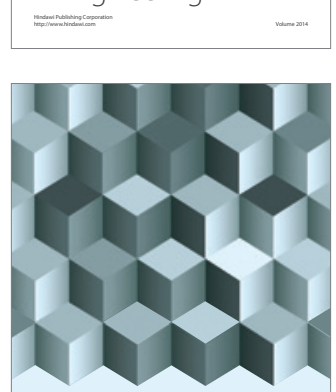

Journal of

Function Spaces
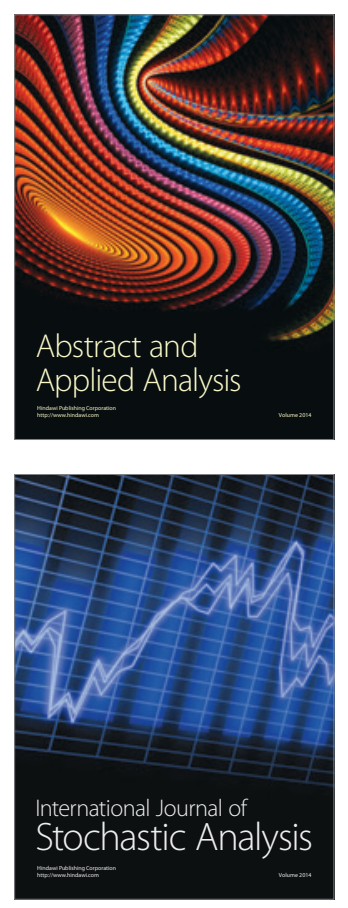

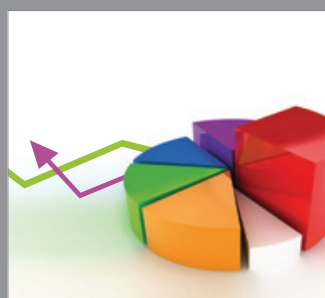

ournal of

Probability and Statistics

Promensencen
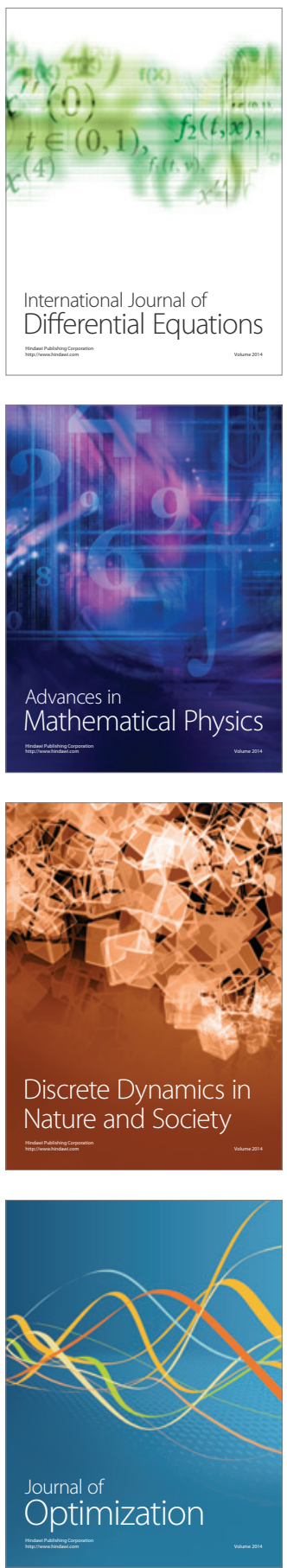